\title{
Perfil de saúde hospitalar dos pacientes submetidos à cirurgia robótica: estudo retrospectivo observacional
}

\author{
Hospital health profile of patients undergoing robotic surgery: a retrospective observational study \\ Perfil de salud hospitalaria de los pacientes sometidos a cirugía robótica: un estudio observacional \\ retrospectivo
}

Recebido: 18/02/2021 | Revisado: 28/02/2021 | Aceito: 05/03/2021 | Publicado: 15/03/2021

Ricardo de Oliveira Meneses
ORCID: https://orcid.org/0000-0001-9962-2827
Universidade do Estado do Rio de Janeiro, Brasil
Instituto Federal de Educação, Ciência e Tecnologia do Rio de Janeiro, Brasil
E-mail: ricardo.meneses@ifrj.edu.br
Letícia Costa Matos
ORCID: https://orcid.org/0000-0002-2447-7327
Universidade do Estado do Rio de Janeiro, Brasil
E-mail: leticiallcmatos@gmail.com
Tatiana de Araujo Eleuterio
ORCID: https://orcid.org/0000-0002-8043-2350
Universidade do Estado do Rio de Janeiro, Brasil
E-mail: tatirodriguesaraujo@yahoo.com.br
Cintia Silva Fassarella
ORCID: https://orcid.org/0000-0002-2946-7312
Universidade do Estado do Rio de Janeiro, Brasil
Universidade do Grande Rio, Brasil
E-mail: cintiafassarella@gmail.com
Denis dos Santos Pinheiro
ORCID: https://orcid.org/0000-0001-9912-8562
Universidade Federal Fluminense, Brasil
E-mail: denispinheiro@id.uff.br
Gisele Passos Cabral Benjamim
ORCID: https://orcid.org/0000-0002-4481-3404
Universidade do Estado do Rio de Janeiro, Brasil
Instituto Nacional de Câncer, Brasil
E-mail giselepcabral@gmail.com

\section{Resumo}

A cirurgia robótica é uma tecnologia minimamente invasiva com a necessidade de treinamento específico e conhecimento da clientela para a operacionalização desse recurso. Faz-se necessário conhecimento profundo dessa realidade e dos fatores determinantes de aspectos clínicos e procedimentais nos hospitais de formação. O estudo trouxe como objetivo identificar o perfil cirúrgico dos pacientes submetidos à cirurgia robótica em um hospital universitário. Como metodologia, trata-se de um estudo retrospectivo, com abordagem quantitativa descritiva. A coleta de dados foi a partir dos registros dos prontuários dos usuários assistidos pela equipe de cirurgia robótica, de julho a dezembro de 2019. Os resultados mostraram que foram realizados 139 procedimentos, sendo a cirurgia urológica a principal especialidade robótica (61,2\%), em homens $(64,7 \%)$ entre 60 e 69 anos $(43,2 \%)$, ASA 2 (77,7\%) e a hipertensão o fator de risco mais frequente $(46,8 \%)$. A cirurgia robótica utiliza a anestesia geral $(91,3 \%) \mathrm{com}$ um tempo médio de 2 a 4 horas de cirurgia $(43,2 \%)$ e 4 a 6 horas sob efeito anestésico $(57,5 \%)$. A posição de Trendelemburg associada à litotomia ocorreu na maioria das cirurgias $(45,3 \%)$ com escala ELPO com escore maior ou igual a $20(52,5 \%)$. A capnografia é fundamental (95,7\%), além do acesso venoso periférico (97,9\%), sendo a complicação perioperatória a via aérea difícil (2,9\%), ainda que os escores baixos estejam de acordo com os dados internacionais. Conclusão: os dados obtidos neste estudo permitiram identificar o perfil dessa clientela, oferecendo embasamento à equipe de saúde para atuar de acordo com a especificidade cirúrgica.

Palavras-chave: Perfil de saúde; Centro cirúrgico hospitalar; Procedimentos cirúrgicos robóticos; Período perioperatório.

\footnotetext{
Abstract

Robotic surgery is a minimally invasive technology with the need for specific training and knowledge of the clientele to operationalize this resource. In-depth knowledge of this reality and the determining factors of clinical and procedural aspects in training hospitals is necessary. The study aimed to identify the surgical profile of patients
} 
undergoing robotic surgery at a university hospital. As a methodology, this is a retrospective study, with a descriptive quantitative approach. Data collection was based on the medical records of users assisted by the robotic surgery team, from July to December 2019. The results showed that 139 procedures were performed, with urological surgery being the main robotic specialty $(61.2 \%)$, in men $(64.7 \%)$ between 60 and 69 years old $(43.2 \%)$, ASA 2 (77.7\%) and hypertension the most frequent risk factor $(46.8 \%)$. Robotic surgery uses general anesthesia $(91.3 \%)$ with an average time of 2 to 4 hours of surgery (43.2\%) and 4 to 6 hours under anesthetic effect $(57.5 \%)$. The Trendelemburg position associated with the lithotomy occurred in most surgeries $(45.3 \%)$ with an ELPO scale with a score greater than or equal to $20(52.5 \%)$. Capnography is essential $(95.7 \%)$ in addition to peripheral venous access (97.9\%), with perioperative complications being the difficult airway (2.9\%), although the scores are low according to international data. Conclusion: The data obtained in this study allowed to identify the profile of this clientele, offering a basis for the health team to act according to the surgical specificity.

Keywords: Health profile; Hospital surgical center; Robotic surgical procedures; Perioperative period.

\section{Resumen}

La cirugía robótica es una tecnología mínimamente invasiva con la necesidad de formación y conocimientos específicos de la clientela para poner en funcionamiento este recurso. Es necesario un conocimiento profundo de esta realidad y de los determinantes de los aspectos clínicos y procedimentales en los hospitales de formación. El estudio tuvo como objetivo identificar el perfil quirúrgico de los pacientes sometidos a cirugía robótica en un hospital universitario. Como metodología, se trata de un estudio retrospectivo, con enfoque descriptivo cuantitativo. La recogida de datos se basó en las historias clínicas de los usuarios atendidos por el equipo de cirugía robótica, de julio a diciembre de 2019.Los resultados mostraron que se realizaron 139 procedimientos, siendo la cirugía urológica la principal especialidad robótica (61,2\%), en hombres (64,7\%). ) entre 60 y 69 años (43,2\%), ASA 2 (77,7\%) e hipertensión el factor de riesgo más frecuente $(46,8 \%)$. La cirugía robótica utiliza anestesia general $(91,3 \%)$ con un tiempo promedio de 2 a 4 horas de cirugía (43,2\%) y de 4 a 6 horas bajo efecto anestésico (57,5\%). La posición de Trendelemburg asociada a la litotomía ocurrió en la mayoría de las cirugías $(45,3 \%)$ con una escala ELPO con una puntuación mayor o igual a $20(52,5 \%)$. La capnografía es fundamental $(95,7 \%)$ además del acceso venoso periférico (97,9\%), siendo las complicaciones perioperatorias la vía aérea difícil (2,9\%), aunque las puntuaciones son bajas según datos internacionales. Conclusión: Los datos obtenidos en este estudio permitieron identificar el perfil de esta clientela, ofreciendo una base para que el equipo de salud actúe de acuerdo con la especificidad quirúrgica.

Palabras clave: Perfil de salud; Centro quirúrgico del hospital; Procedimientos quirúrgicos robóticos; Período perioperatorio.

\section{Introdução}

O conceito de cirurgia robótica vem ao encontro das proposições do que conhecemos como procedimentos cirúrgicos convencionais, pois com o avanço da tecnologia em busca de melhores resultados para um menor estado de comorbidade e promoção de um reduzido trauma operatório os estudos nessa área têm sido aprimorados desde a década de 80 , diante das intervenções cirúrgicas complexas que têm sido base das preocupações interdisciplinares (Skinovsky, Chibata \& Siqueira, 2008).

Pensar nesse procedimento envolve ainda a preocupação com custos elevados e suas implicações com relação à anestesia e ao perfil de pacientes visando melhores resultados aos procedimentos que envolvem diversas especialidades, como cirurgia cardíaca, torácica, geral, urológica, ginecológica e proctológica (Rosa, Moreira \& Leite, 2012).

Atualmente, a cirurgia robótica é conceituada como uma tecnologia minimamente invasiva, que se caracteriza por um dispositivo tecnológico de alta precisão que possui um console localizado à distância do paciente, por onde o cirurgião manipula o instrumental, um carrinho de visão e um robô, composto por quatro braços robóticos, com flexibilidade de 360o. Ainda assim, a equipe de cirurgiões precisa estar apta, caso haja necessidade de conversão do procedimento para uma cirurgia aberta (Carlos \& Saulan, 2018).

Historicamente, sua criação ocorreu na década de 80 devido à necessidade de se realizar procedimentos cirúrgicos em militares nos cenários de guerra, sem colocar em risco a segurança do cirurgião. Os protótipos iniciais ganharam melhores desenvolvimentos a partir dos anos 90, período em que foram desenvolvidos para uso comercial os sistemas cirúrgicos Zeus ${ }^{\circledR}$ e, em 1995, a empresa americana Intuitive Surgical desenvolveu o robô Da Vinci Surgical System®, inserindo a cirurgia robótica como procedimento, pioneiramente nos Estados Unidos e na Bélgica (Rosa, Moreira, Leite \& 2012). Em 2001, 
realizou-se a primeira cirurgia transatlântica, por Marescorix e Gagner, de Nova York em um paciente localizado na França (Skinovsky, Chibata \& Siqueira, 2008). Segundo os dados disponíveis no site da empresa Intuitive ${ }^{\circledR}$, até 2019 estavam cadastrados 5.582 sistemas Da Vinci, distribuídos em 67 países, localizando-se em maior proporção na América do Norte, América Latina e Europa.

No Brasil, a primeira cirurgia robótica ocorreu no ano de 2008 (Rosa, Moreira, Leite \& 2012). Em 2011, em São Paulo, o primeiro hospital público solicitou a incorporação do Sistema Cirúrgico Robótico Da Vinci ao Sistema Único de Saúde (SUS), porém houve posicionamento contrário à adesão desse sistema no país para área pública (Conitec, 2018).

Em 2017, o Programa de Cirurgia Robótica de um hospital privado brasileiro publicou os resultados de sua implementação, evidenciando sua segurança e destacando os benefícios de se utilizar essa abordagem para alguns procedimentos (Madureira et al., 2017).

Em 2019, segundo a empresa Strattner®, havia 74 plataformas em 15 estados brasileiros.

As vantagens desse tipo de abordagem no período perioperatório, quando comparado à cirurgia aberta, incluem: redução do tempo de internação; redução da ocorrência de complicações; menor perda sanguínea; diminuição da dor pósoperatória; melhores resultados estéticos; e redução do tempo de afastamento das atividades diárias (Carlos \& Saulan, 2018).

Já as vantagens para a equipe cirúrgica perioperatória incluem: redução dos tremores, proporcionando maior precisão; promoção da imagem em 3D de alta resolução; melhor estabilidade; maior mobilidade da instrumentação intracorpórea, favorecendo a ergonomia do cirurgião; redução dos riscos de exposição à radiação; menor necessidade de uso de auxiliares; e melhoria do controle do campo operatório (Madureira et al., 2017).

Apesar dos benefícios listados, existem desafios a serem enfrentados, como o custeio público e privado de implementação, a acessibilidade da população e a manutenção do robô. O Sistema Único de Saúde (SUS) não aderiu ao aparelhamento sob a justificativa de que incorporar um sistema de cirurgia robótica representaria a mesma possibilidade de se adquirir dezenas de novos sistemas de videolaparoscopias, que cobririam vazios assistenciais no país, onde não havia ainda a disponibilidade de tais recursos (Conitec, 2018).

A necessidade de treinamento específico para adquirir as habilidades necessárias à operacionalização da técnica se destaca como um dos desafios a serem superados. E por se tratar de uma tecnologia complexa e inovadora, faz-se necessário investir no preparo da equipe, proporcionando treinamentos e conhecimento acerca da clientela que deverá ser assistida. Apropriar-se da realidade dos usuários torna operacionais as necessidades de modificações da rotina perante o uso dessa tecnologia. Uma equipe capacitada e treinada para atuar em cirurgias robóticas é tão necessária quanto os equipamentos específicos (Carlos \& Saulan, 2018).

A maior interface da equipe de saúde com os robôs não será alcançada apenas com novas ferramentas, mas pela integração da inteligência artificial e uma nova maneira de interpretar a cirurgia moderna como uma mudança de paradigma que vale a pena ser seguida (Morrel et al., 2021).

Os principais desafios enfrentados pelo enfermeiro na cirurgia robótica incluem o desenvolvimento de novas competências, a formação de equipe e a segurança do paciente (Pinto et al., 2018). A capacitação de enfermagem para atuar em cirurgia robótica pode solucionar dificuldades diárias com equipamentos ou com os instrumentos do sistema robótico, contribuindo para o sucesso do procedimento.

Para isso, faz-se necessário conhecimento profundo dessa realidade e dos fatores determinantes de aspectos clínicos e procedimentais dessa clientela. Trabalhando com ferramentas da epidemiologia e com indicadores dos serviços de saúde, propôs-se como questão de pesquisa: qual o perfil de saúde dessa clientela que se submete à cirurgia robótica em um hospital universitário?

Considerando a realidade brasileira e o fato de os hospitais universitários serem escolas interdisciplinares de formação 
pública para difusão dessa tecnologia em saúde, os critérios e o impacto a serem pensados na implementação dos serviços robóticos podem surgir a partir do conhecimento sobre a especificidade dessa clientela com a reformulação das práticas avançadas em saúde (Gomes, 2015).

Em uma busca inicial nas bases de dados pela Biblioteca Virtual em Saúde, Scopus e na National Library of Medicine pelo PubMed, identificou-se a escassez de pesquisas publicadas com o propósito deste estudo no país até o momento. Sendo assim, é imprescindível que as unidades de saúde divulguem esse perfil, como justificativa, de modo a estabelecer medidas referentes à organização dessa tecnologia e seus cuidados, enriquecendo o conhecimento acadêmico sobre o tema na área hospitalar.

Diante do exposto, o objetivo do estudo é identificar o perfil cirúrgico dos pacientes submetidos à cirurgia robótica em um hospital universitário.

\section{Metodologia}

Trata-se de um estudo retrospectivo, com abordagem quantitativa descritiva, desenvolvido a partir da coleta de dados obtidos nos prontuários dos usuários assistidos pela equipe de cirurgia robótica do local da pesquisa.

A pesquisa realizou-se em um hospital público universitário, localizado no município do Rio de Janeiro, que é referência em especialidades e importante núcleo nacional de formação de profissionais na área de saúde. O hospital possui cerca de 44 mil m² de área construída e segue em expansão.

O cenário possui atualmente 525 leitos funcionantes e mais de 60 especialidades e subespecialidades. $\mathrm{O}$ hospital não atende emergências abertas e o seu o acesso se dá pelo Sistema de Regulação de Vagas do Estado do Rio de Janeiro. Destacase que a missão da instituição é prestar assistência integrada, humanizada e de excelência à saúde, sendo agente transformador da sociedade por meio do ensino, pesquisa e extensão. Para isso, o hospital mantém programas permanentes de atualização e modernização, por meio de recursos captados com projetos, em especial relacionados à saúde do homem, como também de cirurgia experimental de neofaloplastia, desenvolvidos por profissionais habilitados.

Dentro dos princípios de elegibilidade o universo do foram as especialidades cirúrgicas habilitadas a realizar a cirurgia robótica até a partir do ano de 2019, quando se iniciou o serviço na unidade em 14 de fevereiro. Abrangeram-se as seguintes especialidades habilitadas ao serviço de robótica: urologia, ginecologia, torácica e cirurgia geral. Logo, a amostra incluiu todos os prontuários de pacientes submetidos à cirurgia robótica no ano de 2019, do período da sua implantação até 31 de dezembro.

Como critério de exclusão, adotou-se a indisponibilidade do prontuário, no entanto todos os documentos necessários para a realização deste estudo estavam acessíveis para consulta e observação das informações.

Como critério de exclusão, adotou-se a indisponibilidade do prontuário, no entanto todos os documentos necessários para a realização deste estudo estavam acessíveis para consulta e observação das informações.

Portanto, como cálculo amostral, utilizaram-se $100 \%$ dos prontuários do período do estudo com nível de confiabilidade de $95 \%$ na produção dos dados estatísticos.

A coleta de dados ocorreu de janeiro a junho de 2020 por meio da leitura na íntegra de cada prontuário impresso e digital. Consultaram-se também os registros anestésicos e registros de enfermagem de cada procedimento, em seus respectivos setores, e demais elementos, como as prescrições médicas e registros de enfermagem.

As variáveis foram captadas por meio de um instrumento pré-estruturado, contendo as seguintes informações descritas no prontuário da assistência perioperatória: idade; sexo; índice de massa corporal (IMC); fatores de risco; classificação do estado físico e risco anestésico de acordo com a American Society of Anesthesiologists (ASA); especialidade cirúrgica; tipo de cirurgia; tipo de anestesia; tempo de procedimento; posicionamento cirúrgico; escala de avaliação de risco para o 
desenvolvimento de lesões decorrentes do posicionamento cirúrgico (ELPO); uso de dispositivos invasivos e não invasivos; intercorrências e complicações.

A classificação categórica das variáveis qualitativas foi ordenada de acordo com as referências teóricas aportadas no estudo e as quantitativas, como tempo, foram classificadas pelo porte cirúrgico em intervalo de duas horas de acordo programa operatório em referências nacionais e internacionais.

Por se tratar de um estudo com dados a partir de prontuários e apresentados de forma anônima, não se fez necessário o uso do Termo de Consentimento Livre e Esclarecido - TCLE.

Considerando que toda pesquisa com seres humanos envolve riscos em tipos e gradações variadas, este estudo apresenta risco mínimo relacionado à segurança dos dados confidenciais disponíveis nos prontuários da instituição.

Os dados colhidos foram tabulados por meio do software Microsoft Excel, versão 10, e analisados sob a estatística descritiva por meio do software Statistical Package for the Social Sciences (SPSS), versão 25, com p valor de 0,05.

O estudo foi aprovado pelo Comitê de Ética e Pesquisa (CEP) do hospital em dezembro de 2019, com CAAE: 26299019.6.0000.5259 e parecer: 3.770.845, atendendo às Resoluções 466/12 e 510/16.

\section{Resultados e Discussão}

Realizaram-se 139 procedimentos cirúrgicos robóticos no cenário da pesquisa, no período de julho a dezembro do ano de 2019. As principais características dos pacientes submetidos à cirurgia robótica foram descritas na Tabela 1 .

Tabela 1. Distribuição das características dos pacientes submetidos à cirurgia robótica $(\mathrm{n}=139)$. Rio de Janeiro, RJ, Brasil, 2019.

\begin{tabular}{l|c|c}
\hline Características & $\mathbf{n = 1 3 9}$ & \% \\
\hline Faixa etária (anos) & 17 & 12,2 \\
$19-39$ & 14 & 10,1 \\
$40-49$ & 37 & 26,6 \\
$50-59$ & 60 & 43,2 \\
$60-69$ & 11 & 07,9 \\
$70-79$ & & \\
& & \\
Sexo & 89 & 64,0 \\
Masculino & 49 & 35,3 \\
Feminino & 1 & 0,7 \\
Mulher transexual & & \\
& & \\
Classificação do IMC & 51 & 36,7 \\
Peso normal & 45 & 32,4 \\
Sobrepeso & 28 & 20,1 \\
Obesidade grau 1 & 9 & 6,5 \\
Obesidade grau 2 & 4 & 2,9 \\
Abaixo do peso & 2 & 1,4 \\
Obesidade grau 3 & & \\
& & \\
& & \\
\hline
\end{tabular}




\begin{tabular}{l|c|c}
\hline Fatores de risco & & \\
Hipertensão & 65 & 46,8 \\
Tabagismo & 19 & 13,7 \\
Diabetes mellitus & 12 & 8,6 \\
Asma & 6 & 4,3 \\
Dislipidemia & 3 & 2,2 \\
DPOC & 2 & 1,4 \\
Tuberculose & 1 & 0,7 \\
Febre & 1 & 0,7 \\
Bronquite & 1 & 0,7 \\
& & \\
ASA & & 19,4 \\
1 & 27 & 77,7 \\
2 & 108 & 2,9 \\
3
\end{tabular}

Fonte: Autores (2020).

A idade dos pacientes variou de 19 a 73 anos, sendo 60 a 69 anos o intervalo etário mais frequente, com 43,2\%, e como outlier a idade de 19 anos, com menos que 1\%. O maior grupo de pacientes era do sexo masculino com 64\%, sendo $35,3 \%$ do sexo feminino e uma mulher transexual, com $0,7 \%$, devido à unidade ser referência em redesignação sexual sob carácter experimental no país.

Com relação ao índice de massa corporal (IMC), 36,7\% dos pacientes tinham peso normal, 32.4\% estavam com sobrepeso, $28 \%$ eram obesos e 2,9\% estavam abaixo do peso. O índice de massa corporal foi avaliado em razão de a obesidade ser considerada um fator de alto risco para desenvolvimento de complicações cirúrgicas, como lesões por posicionamento cirúrgico, ainda que os maiores riscos do baixo peso e obesidade grau 3 sejam os que trazem maior preocupação a esse tipo de procedimento pelo risco de lesão por posicionamento e risco hemodinâmico (Coelho, 2016).

Como resultado dos fatores de risco do estudo, a hipertensão abrangeu 46,8\%, seguida do tabagismo, com 13,7\%, e diabetes mellitus, com 8,6\%, tendo a clientela índices menores que 5\% nos demais fatores, como a asma, a dislipidemia, a doença pulmonar obstrutiva crônica, a tuberculose e a febre e a bronquite.

Ao analisar na literatura os fatores de risco a serem considerados em pacientes que são submetidos à cirurgia robótica, destacaram-se as doenças cardiovasculares, neurológicas, vasculares e pulmonares preexistentes, comparando-se com os dados deste estudo. Logo, a hipertensão apresentou-se como principal fator de risco, corroborando com perfil nacional de acometimento de doenças de base a serem considerados no perfil de saúde desses usuários (Esqueda et al., 2020).

Em virtude da adoção do posicionamento íngreme de Trendelemburg, os pacientes com doença pulmonar obstrutiva crônica (DPOC) tendem a não recuperar totalmente os parâmetros dos sinais vitais que se alteram durante o procedimento. Devido a isso, a DPOC é considerada uma contraindicação para a realização da cirurgia robótica, assim como o enfisema pulmonar (Maerz et al., 2017). Contudo, neste estudo, os seguintes fatores de risco pulmonares foram identificados: 13,7\% dos pacientes eram tabagistas, 4,3\% asmáticos, 1,4\% possuíam DPOC e 0,7\% foi descrito a bronquite, estando em acordo com adesão de escolha para este tipo de procedimento com baixo percentual frente ao perfil de risco relacionado a cirurgia robótica.

Além disso, pacientes portadores de glaucoma apresentam contraindicação para realização de cirurgias robóticas que necessitam do posicionamento íngreme de Trendelemburg, por ocorrer aumento da pressão intraocular, podendo causar 
isquemia do nervo óptico e alterações da função visual. Neste estudo, não identificamos nos registros pacientes portadores de glaucoma (Esqueda et al., 2020).

Além dos já citados, os outros fatores de risco o diabetes $(8,6 \%)$ que devem ser considerados como aspectos relevantes para a ocorrência de complicações vasculares relacionadas ao posicionamento citado.

Em um estudo de revisão apresentou que os eventos cardíacos decorrentes de complicações são baixos em 1\% de uma amostra de 182 usuários submetidos a cirurgia laparoscópica. Observou-se que desenvolveram fibrilação atrial com alterações significativas no eletrocardiograma resultado em congestão pulmonar. Desta forma aspectos no mapeamento dos fatores de risco são fundamentais (Maerz et al., 2017)

Um dado importante para a decisão anestésica é a classificação de estado físico e risco anestésico estabelecido pela American Society of Anesthesiologists (ASA), conforme variável categórica apresentada na tabela 1. Neste estudo, 77,7\% dos pacientes eram ASA 2 que indica discreta limitação sistêmica ou doença controlada; 19,4\% eram ASA 1 que classifica os pacientes sem riscos ou normais e 2,9\% eram ASA 3, compreendidos como doença limitante.

Apesar dos pacientes classificados como ASA 1 e ASA 2, que representam a maioria dos pacientes assistidos no cenário investigado com 97,1\%, no que se trata das condições físicas mais estáveis e menores riscos relacionados ao procedimento anestésico, existe a possibilidade de complicações cardiovasculares relacionadas ao posicionamento de Trendelenburg. Desta forma, os dados do estudo convergem que a classificação anestésica dos pacientes da cirurgia robótica devem ser menores e são de acordo com perfil perioperatório estimado, considerando a classificação dos quadros clínicos associarem-se a própria patologia de base dos usuários (Coelho, 2016).

Em próximo item serão apresentados o perfil cirúrgico da cirurgia robótica apresentando conforme Tabela 2.

Tabela 2. Perfil dos pacientes submetidos à cirurgia robótica no centro cirúrgico hospitalar (n=139). Rio de Janeiro, RJ, Brasil, 2019.

\begin{tabular}{l|l|l}
\hline Característica & $\mathbf{n}=\mathbf{1 3 9}$ & $\%$ \\
\hline Especialidade cirúrgica & 85 & 61,2 \\
Cirurgia urológica & 23 & 16,5 \\
Cirurgia ginecológica & 16 & 11,5 \\
Cirurgia geral & 15 & 10,8 \\
Cirurgia torácica & & \\
& & \\
Anestesia & 127 & 91,3 \\
Geral & 8 & 5,8 \\
Combinada & 4 & 2,9 \\
Geral + bloqueio após o fim do procedimento & \\
Tempo de cirurgia (horas) & 60 & 43,2 \\
$>2-4$ & 52 & 37,4 \\
$>4-6$ & 14 & 10,1 \\
$>1-2$ & 12 & 8,6 \\
$>6$ & 1 & 0,7 \\
Até 1 & &
\end{tabular}




\begin{tabular}{l|l|l}
\hline Tempo de anestesia (horas) & 80 & 57,5 \\
$>4-6$ & 30 & 21,6 \\
$>2-4$ & 29 & 20,9 \\
$>6$ & & \\
Posicionamento & 63 & 45,3 \\
Trendelemburg + litotômica & 38 & 27,3 \\
Dorsal & 24 & 17,3 \\
Lateral & 5 & 3,6 \\
Anti-Trendelemburg & 5 & 3,6 \\
Trendelemburg & 3 & 2,2 \\
Ignorada & 1 & 0,7 \\
Litotômica & & \\
& & \\
Sgnorado & 63 & 52,5 \\
\hline
\end{tabular}

Fonte: Autores (2020).

De acordo com os dados da Tabela 2, referente às variáveis das especialidades dos pacientes submetidos à cirurgia robótica, a maioria deles foi submetida aos procedimentos urológicos, com $61,2 \%$, seguidos da cirurgia ginecológica, com $16,5 \%$, cirurgia geral, com $11,5 \%$, e cirurgia torácica, com $10,8 \%$.

Faz-se necessário citar que alguns fatores interferem na decisão de utilização do sistema robótico pelas especialidades cirúrgicas. Como a disponibilidade da sala para utilização do sistema robótico, o número de cirurgiões habilitados para realização de cirurgia robótica varia de acordo com cada clínica, assim como a disponibilidade de pacientes que se enquadrem no perfil necessário que o torne apto a ser submetido a um procedimento robótico.

Quanto aos procedimentos, a cirurgia realizada com maior frequência foi a prostatectomia radical, com 48,2\% ( $\mathrm{n}=67$ ), seguida pela nefrectomia, com 8,6\% $(\mathrm{n}=12)$, dentro da especialidade urologia, corroborando com o relatório da Conitec (2018) que aponta a prostatectomia radical para tratamento do câncer de próstata como a intervenção cirúrgica mais realizada no Brasil e em outros países nas cirurgias robóticas.

Apesar de ser considerada padrão-ouro no tratamento do câncer de próstata, a prostatectomia radical não robótica é responsável por complicações em longo prazo, como a incontinência urinária, a disfunção erétil e, por consequência, alterações psicossociais, como ansiedade, depressão e baixa da autoestima (Brasil, 2015). Dada a preservação das funções urinária e sexual nesse tipo de abordagem, a cirurgia robótica de próstata surge como uma alternativa, tornando o procedimento mais preciso.

No que se refere à duração dos procedimentos, a maioria $(43,2 \%)$ foi realizada entre 2 e 4 horas, classificada como porte II no que se refere ao tempo cirúrgico. O tempo em cirurgia no console é um componente de avaliação em qualquer processo de treinamento e habilitação em cirurgia robótica. Considerando a curva de aprendizagem, espera-se que quanto maior a experiência, menor o tempo de console. Quanto menor o tempo de procedimento, menor o tempo de anestesia. Neste estudo, a maioria dos pacientes ficou submetida à anestesia por um período de 4 a 6 horas (57,5\%), mostrando que o tempo de intervenção anestésica pode agregar maior fator de risco que a cirurgia (Nacul et al., 2020). 
Com relação à modalidade anestésica utilizada de acordo com a Tabela 2, a maior parte dos pacientes recebeu anestesia geral, com 91,3\%, seguida, em menor proporção, da anestesia combinada e geral com bloqueio após o fim do procedimento, com $8,7 \%$.

A combinação da anestesia geral com um bloqueio regional auxilia na ventilação e oxigenação durante o procedimento e no controle da dor pós-operatória. A escolha anestésica para cirurgia robótica é a anestesia geral com ventilação mecânica, associada a um bom relaxamento muscular, favorecendo também a introdução do equipamento cirúrgico, ainda que a maioria seja urológica, pois o pneumoperitônio também contribui para isso, corroborando com os dados deste estudo (Coelho, 2016).

Em um estudo observacional retrospectivo em comparação com o manejo anestésico entre cirurgias laparoscópicas e robóticas de prostatectomia, os pacientes receberam anestesia balanceada geral, corroborando os dados do perfil de pacientes investigados (Yokura, 2016).

Outro dado apresentado na Tabela 2, o posicionamento cirúrgico, 45,3\% dos pacientes foram posicionados em Trendelemburg severo com litotomia, sendo essa a posição mais utilizada. Alguns dos procedimentos que utilizam esse posicionamento cirúrgico são as prostatectomias radicais, corroborando com Yokura (2016), cistectomias, histerectomias e ressecção dos focos de endometriose.

Apesar de ser responsabilidade de todos da equipe e de se tratar de um procedimento que envolve riscos, em 2,2\% dos procedimentos não foram registradas tais informações nos documentos analisados. Ao realizar o posicionamento cirúrgico do paciente no período intraoperatório, é preciso ter como objetivo a segurança, o conforto, a prevenção de complicações e o acesso necessário à assistência anestésica, além da preferência do cirurgião, de acordo com o sítio cirúrgico. A atenção inadequada ao posicionamento e à falta de uso de dispositivos auxiliares é diretamente atribuída a lesões de posicionamento (Maerz et al., 2017).

A associação entre posicionamento íngreme de Trendelemburg e o aumento da pressão intra-abdominal é em razão do pneumoperitônio e do tempo cirúrgico prolongado, que podem levar a complicações graves. As mais comuns incluem a atelectasia grave, o aumento do nível de $\mathrm{PaCO}$, a acidose, o edema pulmonar e a perda residual moderada da complacência pulmonar.

Tais complicações podem ser bem toleradas por pacientes sem comorbidades pulmonares, mas são significativas quando há presença de patologias respiratórias. Contudo, o aumento da pressão das vias aéreas é inevitável, principalmente quando adotado o posicionamento íngreme de Trendelemburg (Yetim, Tekindur \& Kilickaya, 2017).

Nesse sentido, a Escala de Avaliação de Risco para o Desenvolvimento de Lesões Decorrentes do Posicionamento Cirúrgico (ELPO) pode ser utilizada com o intuito de identificar os pacientes que possuem maior chance de apresentar dor ou desenvolver lesões no período pós-operatório por conta do posicionamento cirúrgico. Dessa forma, é possível que o profissional desenvolva suas ações de forma planejada para promoção do alívio de pressão, diminuindo, assim, o risco de o paciente ser acometido por lesões decorrentes do posicionamento cirúrgico (Lopes et al., 2016).

Em 52,5\% das cirurgias robóticas realizadas, o escore ELPO foi igual ou maior a 20, apontando para maior risco de desenvolvimento de lesões. Ainda assim, não foram registrados casos de lesões por posicionamento cirúrgico que pudessem ter sido identificados nos registros posteriores à intervenção. No país, esse processo de implantação é novo, visto o tempo de produção dessa tecnologia e o investimento em materiais de viscoelásticos na posição requerida pela robótica, sendo um dado a ser considerado na adoção desse recurso para qualidade da assistência prestada (Souza et al., 2018).

Ao realizar o levantamento dos dispositivos utilizados para avaliação e manutenção dos parâmetros fisiológicos, bem como os equipamentos acoplados ao paciente conforme Tabela 3, no intraoperatório, observou-se que a carência dos registros acerca de um dispositivo pode não significar que o mesmo não tenha sido utilizado, mas, sim, uma fragilidade com relação aos 
dados informados nos documentos. No mais, considerando que todas as cirurgias robóticas no centro cirúrgico da unidade estudada utilizam placa dispersiva, oxímetro e monitorização cardíaca, tais dispositivos não foram contabilizados por estarem presentes em $100 \%$ dos procedimentos.

Tabela 3. Dispositivos utilizados no paciente durante o intraoperatório no centro cirúrgico hospitalar. Rio de Janeiro, RJ, Brasil, 2019.

\begin{tabular}{l|c|c}
\hline Dispositivos & $\mathbf{n}$ & $\%$ \\
\hline Não invasivos & 133 & 95,7 \\
Capnógrafo & 132 & 95 \\
Índice bispectral & 124 & 89,2 \\
Pressão arterial sistêmica & 106 & 76,3 \\
Train-of-four & 82 & 59 \\
Faixa de segurança & 71 & 51,1 \\
Termômetro & 39 & 28,1 \\
Coxins & 30 & 21,6 \\
Manta térmica & 2 & 1,4 \\
Perneira pneumática & 1 & 0,7 \\
Meias compressivas & & \\
& & \\
Invasivos & 126 & 90,6 \\
Cateterismo vesical de demora & 102 & 73,4 \\
Acesso venoso periférico bilateral & 74 & 53,2 \\
Drenos & 34 & 24,5 \\
Acesso venoso periférico unilateral & 31 & 22,3 \\
Pressão arterial média & 15 & 10,8 \\
Acesso venoso profundo & & 1,4 \\
Sonda endogástrica & & \\
Cateter peridural & & \\
\hline & & \\
\hline
\end{tabular}

Fonte: Autores (2020).

A fim de obter parâmetros vitais com maior segurança e fidedignidade, os dispositivos podem ser invasivos e não invasivos, variando de acordo com as necessidades de cada paciente (Esqueda et al., 2020). A impossibilidade de movimentação do paciente após o docking é uma das maiores preocupações do anestesista. Desse modo, faz-se necessário garantir que o paciente esteja monitorizado e com os dispositivos necessários para manutenção dos parâmetros vitais, de forma adequada e segura. Da mesma forma, em casos de urgência em que seja preciso acessar ou mobilizar o paciente, a intervenção só será iniciada após realizar o undocking, retardando a assistência (Coelho, 2016).

Os dispositivos para monitorização não invasivos observados nesta pesquisa e apresentados na Tabela 3 estão de acordo com o que a literatura indica (Esqueda et al., 2020). Diversos dispositivos podem ser utilizados com o intuito de garantir a imobilidade do paciente na mesa de cirurgia, auxiliar no posicionamento, bem como evitar lesões.

Neste estudo, o uso de faixas de segurança foi registrado em $59 \%$ dos procedimentos e os coxins em $28,1 \%$. A unidade analisada apresenta coxins de polímero e espuma, mesa cirúrgica com colchões de espuma e diversos posicionadores, incluindo o colchão de vácuo que se adapta ao corpo do paciente e torna-se rígido, sendo um aliado na prevenção das lesões 
por posicionamento cirúrgico. Apesar disso, os registros não informaram os locais onde tais coxins foram utilizados e não descreveram se o colchão de vácuo foi utilizado.

No que tange à manutenção dos parâmetros vitais, o acesso venoso periférico bilateral foi registrado em 73,4\%. Tal conduta é feita por segurança, caso algum dos acessos fique comprometido. Da mesma forma, pacientes com maiores riscos devem ser monitorizados hemodinamicamente com acesso venoso profundo e pressão arterial invasiva de forma mais incisiva devido ao elevado grau de risco correlacionado aos aspectos clínicos (Coelho, 2016).

A sonda endogástrica foi utilizada em $1,4 \%$ dos procedimentos com o intuito de auxiliar o cirurgião durante o procedimento, promovendo o esvaziamento gástrico e melhorando o campo de visão.

Com relação às complicações perioperatórias, este estudo identificou o registro de 20 ocorrências dos 139 investigados, conforme descrito na Tabela 4.

Tabela 4. Complicações perioperatórias registradas em pacientes submetidos à cirurgia robótica $(n=139)$. Rio de Janeiro, RJ, Brasil, 2019.

\begin{tabular}{l|c|c}
\hline Complicações & $\mathbf{n = 1 3 9}$ & $\mathbf{\%}$ \\
Via aérea difícil & 4 & 2,9 \\
Relacionadas ao sistema Da Vinci & 3 & 2,1 \\
Enfisema subcutâneo & 2 & 1,4 \\
Extubação acidental & 2 & 1,4 \\
Conversão para cirurgia aberta & 2 & 1,4 \\
Trauma dentário & 1 & 0,7 \\
Taquicardia & 1 & 0,7 \\
Broncoespasmos & 1 & 0,7 \\
Laceração esplênica & 1 & 0,7 \\
Acidose metabólica & 1 & 0,7 \\
Descompensação cardiológica & 1 & 0,7 \\
Edema facial e em região genital & 1 & 0,7 \\
\hline
\end{tabular}

Fonte: Autores (2020).

De acordo com os dados da Tabela 4, a complicação mais frequente estava relacionada à via aérea difícil (VAD) durante a intubação, tendo ocorrido em $2,9 \%$ dos casos, apesar desse achado não ser descrito com frequência nos estudos (Maerz et al., 2017). Destaca-se que a intubação ocorre antes de ser adotado o posicionamento cirúrgico e não possui especificidades ao ser realizada para um procedimento cirúrgico robótico.

Apesar de ser considerado um fator de risco para trauma dentário, achado relatado em 0,7\% dos registros, sua ocorrência não foi associada à via aérea difícil (VAD). Algumas das possíveis causas podem incluir patologias dentárias preexistentes, anatomia do paciente, qualidade dos equipamentos disponíveis e inabilidade do profissional (Sousa \& Mourão, 2015).

Ainda relacionado ao acesso à via aérea superior, a extubação acidental ocorreu em 1,4\% dos casos, não tendo sido descrito nos registros se ocorreu devido às especificidades da cirurgia robótica.

A segunda complicação mais frequente foi com relação aos incidentes relacionados aos equipamentos $(2,1 \%)$; em 0,7\% ocorreu o acionamento do bisturi elétrico de forma involuntária ao comando do cirurgião; em 0,7\% houve queda de luz; e em $0,7 \%$ foi necessário realizar a troca do portal do trocater. Contudo, nenhuma das complicações relacionadas aos equipamentos levou a complicações correspondentes ou danos ao paciente decorrentes desses imprevistos. 
O estudo de Maerz et al. (2017) aponta que apesar do sistema Da Vinci possuir diversos recursos de segurança podem ocorrer problemas técnicos que coloquem em risco a segurança do paciente, concordando com os dados deste estudo, apesar de não apresentarem associação estatisticamente significativa. Isso pode estar associado ao fato de o apoio técnico da empresa fabricante possuir interação ao vivo com a equipe de forma online, independentemente da localização geográfica em que o procedimento esteja acontecendo (Pinto et al., 2018).

O enfisema subcutâneo ocorreu em 1,4\% dos casos. Tal complicação está relacionada ao uso do pneumoperitônio, sendo considerada um achado comum, apresentando prevalências que variam de $0,3 \%$ a 3,9\%. Apesar de ser transitória, essa complicação pode levar à hipercapnia severa ou cursar com pneumotórax, pneumomediastino ou pneumopericárdio (Coelho, 2016).

Das demais complicações registradas, $2,8 \%$ podem estar relacionadas à habilidade dos cirurgiões, são elas: a conversão para cirurgia aberta, que ocorreu em $2,1 \%$ dos casos, e a laceração esplênica, em $0,7 \%$ dos procedimentos. Um cirurgião que tenha realizado menos de 20 procedimentos cirúrgicos robóticos está no início da curva de aprendizagem do método (Ramos et al. 2013). Todavia, nesses casos é necessária a presença de um cirurgião proctor que deve estar devidamente habilitado em cirurgia robótica e possuir experiência mínima de 35 a 50 procedimentos robóticos (Nacul et al., 2020).

O edema facial e em bolsa escrotal esteve presente em $0,7 \%$ dos casos. Estudos apontam o edema como uma das complicações mais comuns, assim como as neuropatias periféricas, abrasões da córnea e complicações vasculares (Maerz et al., 2017).

Apenas um procedimento foi suspenso devido a uma descompensação cardiológica durante a indução anestésica, que impossibilitou a realização da cirurgia. Também em apenas uma ocorrência o uso de ventilação mecânica para outra unidade foi registrado relacionado com acidose metabólica e dependência de aminas vasoativas.

Em um estudo de revisão realizado em intervenções urológicas robóticas e laparoscópicas de complicações referentes à anestesia, apontou-se que a estimulação vagal pode causar bradiarritmia, assistolia e hipotensão. A taquiarritmia também pode ocorrer como resultado da ativação simpática causada pela hipercarpnia característica do procedimento anestésico. Nesse contexto, as situações de emergências podem ser esperadas necessitando do manejo adequado da equipe de anestesiologistas (Arslan, 2018).

\section{Conclusão}

As variáveis analisadas destacaram que o perfil predominante foi de indivíduos entre 60 e 69 anos, do sexo masculino, com IMC dentro da classificação normal e hipertensão como o fator de risco mais frequente, além da classificação ASA estar entre 1 e 2 pelos riscos anestésicos levados em consideração nas publicações nacionais e internacionais.

O perfil cirúrgico evidenciou a cirurgia urológica como a especialidade predominante, corroborando com dados históricos e atuais no cenário hospitalar, sendo os pacientes submetidos, majoritariamente, à anestesia geral com porte II ( 2 a 4 horas de procedimento) e com 4 a 6 horas sob efeito da anestesia.

A posição de Trendelemburg associado à litotomia ocorreu na maioria das cirurgias robóticas, apresentando a escala de lesão por posicionamento intraoperatório (ELPO) com escore maior ou igual a 20 na maioria dos casos, agregando-se aos riscos anestésicos que apontam o edema de face, lesão ocular, dentre outras preocupações clínicas agravadas por essa postura.

A capnografia precisa ser adotada como tecnologia não invasiva considerando o pneumoperitônio e o posicionamento, além do acesso venoso periférico, como recurso invasivo que promove a intervenção da própria anestesia geral e a terapêutica de controle hemodinâmico por meio desse dispositivo.

A complicação perioperatória registrada com maior frequência foi a via aérea difícil ainda que os escores do estudo tenham sido baixos de acordo com os dados internacionais e os de não ocorrências maiores, conforme registros dos prontuários 
investigados.

Tais dados oferecem maior embasamento à equipe de saúde para atuar na assistência direcionada a esse perfil de saúde dessa clientela, de acordo com a especificidade cirúrgica. Dessa forma, é possível intervir no processo saúde-doença na proposição do planejamento, execução e avaliação das ações de prevenção, controle e tratamento de agravos, bem como fornecer subsídios para estabelecer prioridades na assistência perioperatória dos cenários hospitalares.

Faz-se necessário que outros estudos observacionais retrospectivos no país possam subsidiar dados do perfil cirúrgico desses pacientes, tendo em vista que os impactos da cirurgia robótica nos serviços públicos e privados possam representar uma associação direta com avanço do mundo pós-moderno na gestão dos serviços cirúrgicos e da integralidade do cuidado com menores danos.

As limitações do estudo envolveram as fragilidades com relação à qualidade dos registros por se tratar de coleta de dados secundários. Vale ressaltar que alguns dos documentos físicos consultados para a realização deste estudo não possuíam informações claras e completas a respeito das variáveis. Dessa forma, a informação ignorada acerca de uma variável pode não refletir sua ausência de fato, mas, sim, a incompletude dos registros disponíveis.

Precisa-se atuar nos fatores que dificultam o preenchimento adequado dos registros, bem como investir em educação em serviço, a fim de melhorar a qualidade assistencial. Dessa forma, os dados deste estudo não devem ser generalizáveis, no tocante às peculiaridades do cenário, e podem ter continuidade em futuras pesquisas.

\section{Referências}

Arslan, M. E., \& Özgök, A. (2018). Complications of robotic and laparoscopic urologic surgery relevant to anesthesia. Mini-invasive Surgery, 2(3), 4. https://doi.org/10.20517/2574-1225.2017.31

Carlos, G. \& Saulan, M. (2018) Robotic Emergencies: Are You Prepared for a Disaster? AORN, 108(5), 493-501. 10.1002/aorn.12393.

Coelho, B. A. C. (2016) Revisão de Procedimentos Anestésicos para Prostatectomia Radical Robótica. [Dissertação de Mestrado em medicina]. Faculdade de Medicina da Universidade de Lisboa. https://repositorio.ul.pt/bitstream/10451/26256/1/BeatrizACCoelho.pdf

CONITEC (2018) Relatório de recomendação: Sistema cirúrgico robótico para cirurgia minimamente invasiva: Prostatectomia radical, 2018. http://conitec.gov.br/images/Relatorios/2018/Recomendacao/Relatorio_DaVinci_Prostatectomia.pdf

Esqueda, S. D. et al. (2020) Prostatectomía radical robótica: una revisión de aspectos anestésicos. An Med (Mex), 65(2), 122-129. https://dx.doi.org/10.35366/94365.

Lopes, C. M. M., Haas, V. J., Dantas, R. A. S., Oliveira, C. G., \& Galvão, C. M. (2016). Assessment scale of risk for surgical positioning injuries. Revista Latino-Americana de Enfermagem, 24, e2704. https://doi.org/10.1590/1518-8345.0644.2704

Madureira, F. A. V., Varela, J. L. S., Madureira Filho, D., D'Almeida, L. A. V., Madureira, F. A. V., Duarte, A. M., Vaz, O. P., \& Ramos, J. R. (2017). Model of a training program in robotic surgery and its initial results. Revista do Colégio Brasileiro de Cirurgiões, 44(3), 302-307. https://doi.org/10.1590/010069912017003013

Ministério da Saúde. (2015). Diretrizes Diagnósticas e Terapêuticas do Adenocarcinoma de Próstata. http://conitec.gov.br/images/Consultas/Relatorios/2015/DDT_Adenocarcinomadeprostata_CP.pdf

Morrell, A. L. G., Morrell-Junior, A. C., Morrell, A. G., Mendes, J. M. F., Tustumi, F., De-Oliveira-E-Silva, L. G., \& Morrell, A. (2021). The history of robotic surgery and its evolution: when illusion becomes reality. Revista do Colégio Brasileiro de Cirurgiões, 48, e20202798. https://doi.org/10.1590/01006991e-20202798.

Maerz, D. A., Beck, L. N., Sim, A. J., \& Gainsburg, D. M. (2017). Complications of robotic-assisted laparoscopic surgery distant from the surgical site. British Journal of Anaesthesia, 118(4), 492-503. https://doi.org/10.1093/bja/aex003

Nacul, M. P., Melani, A. G. F., Zilberstein, B., Benevenuto, D. S., Cavazzola, L. T., Araujo, R. L.C., Sallum, R. A. A., Aguiar-Jr, S., \& Tomasich, F. (2020). Educational note: teaching and training in robotic surgery. An opinion of the Minimally Invasive and Robotic Surgery Committee of the Brazilian College of Surgeons. Revista do Colégio Brasileiro de Cirurgiões, 47, e20202681. https://doi.org/10.1590/0100-6991e-20202681

Pinto, E., Lunardi, L., Treviso, P., \& Botene, D. (2018). Atuação do enfermeiro na cirurgia robótica: desafios e perspectivas . Revista SOBECC, 23(1), 43-51. https://doi.org/10.5327/Z1414-4425201800010008

Ramos, A. C., Domene, C. E., Volpe, P., Pajecki, D., D'Almeida, L. A. V., Ramos, M. G., Bastos, E. L. S, \& Kim, K. C. (2013). Resultados iniciais da primeira série de casos brasileira de cirurgia bariátrica totalmente robótica. ABCD. Arquivos Brasileiros de Cirurgia Digestiva, 26(1), 2-7. https://doi.org/10.1590/S0102-67202013000600002 
Research, Society and Development, v. 10, n. 3, e23310313092, 2021

(CC BY 4.0) | ISSN 2525-3409 | DOI: http://dx.doi.org/10.33448/rsd-v10i3.13092

Rosa, M. F., Moreira, M. S., \& Leite, J. B. A. (2012) Aplicação da Robótica nos Centros Cirúrgicos. Instituto Nacional de Telecomunicações, MG. https://www.inatel.br/ic/docman/artigos-publicados-s763609-1/biomedica-s418539-1

Skinovsky, J., Chibata, M., \& Siqueira, D. E. D. (2008) Realidade virtual e robótica em cirurgia - aonde chegamos e para onde vamos? Revista do Colégio Brasileiro de Cirurgiões, 35(5), 334-337. https://doi.org/10.1590/S0100-69912008000500011.

Sousa, C., Bispo, D., \& Acunã, A. (2018). Criação de um manual para posicionamento cirúrgico: relato de experiência. Revista SOBECC, 23(3), 169-175. https://doi.org/10.5327/Z1414-4425201800030009.

Sousa, J. M. B. R., \& Mourão, J. I. B. (2015). Lesão dentária na anestesiologia. Rev. Bras. Anestesiol, 65(6), 511-518. https://doi.org/10.1016/j.bjan.2013.04.009.

Yonekura, H., Hirate, H., \& Sobue, K. (2016). Comparison of anesthetic management and outcomes of robot-assisted vs pure laparoscopic radical prostatectomy. Journal of Clinical Anesthesia, 35, 281-286. https://doi.org/10.1016/j.jclinane.2016.08.014.

Yetim, Memduh, Tekindur, Sukru, \& Kilickaya, Oguz. (2017). Preventing atelectasia at robotic surgery. Revista Brasileira de Anestesiologia, 67(1), 112-113. https://doi.org/10.1016/j.bjane.2015.04.004. 